\title{
Oral Tuberculosis: An Acid Fast Negative Case Report
}

\author{
Mehdi Nasr Isfahani ${ }^{1}$, Parichehr Ghalayani ${ }^{2}$, Fahimeh Pakravan ${ }^{2, *}$ \\ ${ }^{1}$ Department of emergency medicine, Isfahan university of medical sciences, Isfahan, Iran \\ ${ }^{2}$ Department of oral medicine, Isfahan university of medical sciences, Isfahan, Iran \\ *Corresponding author: Pakravanfahimeh@yahoo.com
}

Received May 03, 2014; Revised May 24, 2014; Accepted June 11, 2014

\begin{abstract}
We report a rare case of tuberculosis of oral cavity in a 3.5 year-old male who was referred to oral medicine department with a chief complaint of a painless lesion in the oral cavity. Incisional biopsy from the lesion margin depicted histological features of necrotizing granulomatous lesion. Ziehl-Neelsen staining for acid fast bacilli, interestingly, was negative.
\end{abstract}

Keywords: oral tuberculosis, Ziehl-Neelsen, Acid-fast bacilli

Cite This Article: Mehdi Nasr Isfahani, Parichehr Ghalayani, and Fahimeh Pakravan, "Oral Tuberculosis: An Acid Fast Negative Case Report.” American Journal of Infectious Diseases and Microbiology, vol. 2, no. 3 (2014): 45-47. doi: 10.12691/ajidm-2-3-1.

\section{Introduction}

Tuberculosis (TB) is one of the chronic granulomatous lesions affecting various body systems. It is usually caused by Mycobacterium tuberculosis, although Mycobacterium bovis, Mycobacterium kansasii and Mycobacterium scrofulaceum have also been implicated [1] .Tuberculosis primarily affects the pulmonary site; however, there has been an increased incidence of extrapulmonary sites being affected as well.Oral tuberculosis lesions are infrequent and it is estimated that only $0.05-5 \%$ of total tuberculosis cases may be presented with oral manifestations. Clinically, oral tuberculosis may present as an ulcer, erythematous patches, granulomas, salivary gland involvement, tuberculous lymphadenitis or tuberculous osteomyelitis of the jaws [2].

The aim of this article is to report a case of primary tuberculosis and to emphasize the importance of early diagnosis with various diagnostic tests so as to lessen the risk of exposure to an infected patient.

\section{Case Report}

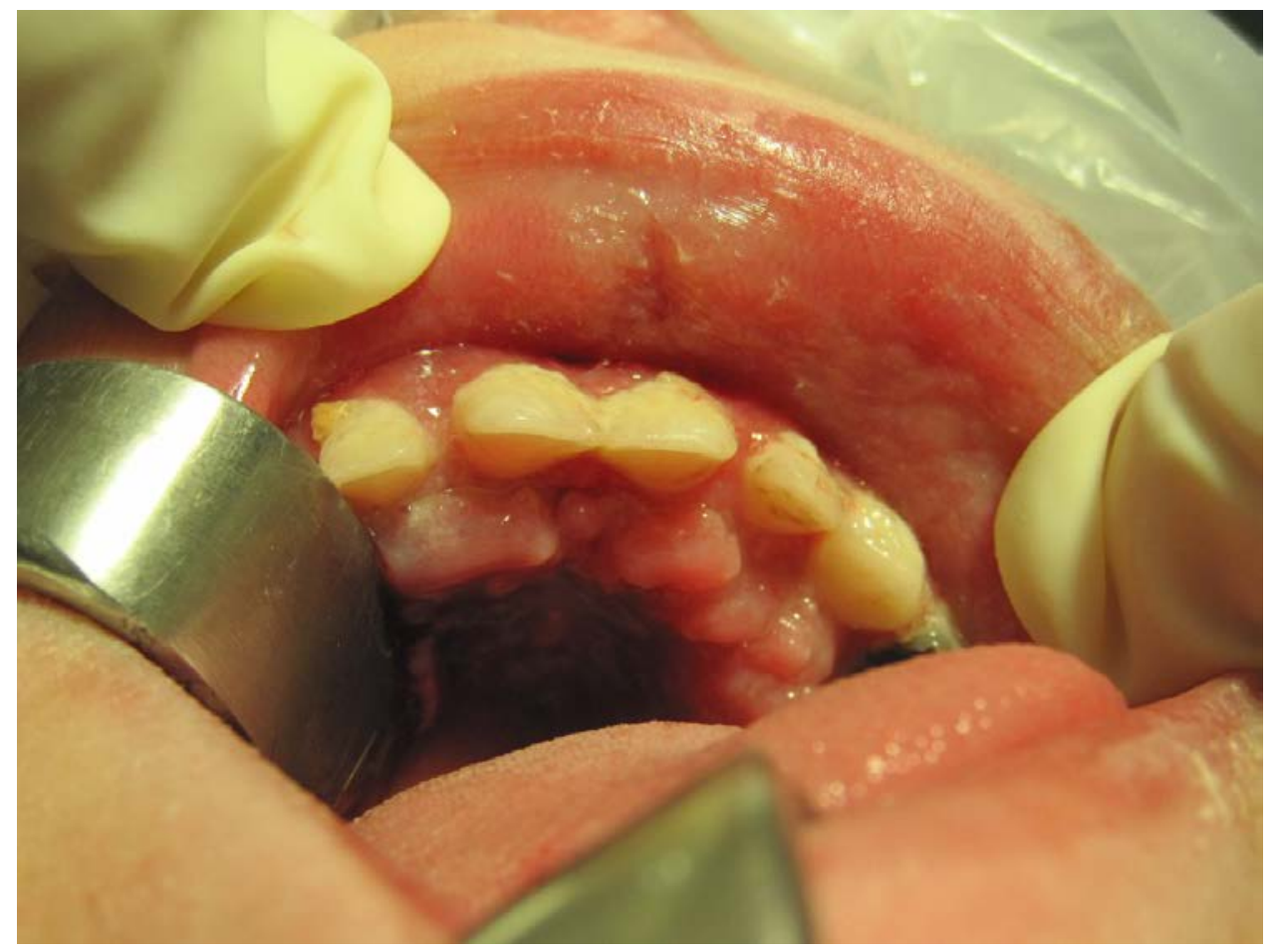

Figure 1. 
A 3.5 year old boy was brought to the oral medicine department of our hospital from a rural area with complaints of a painless, non-healing lesion, in the oral cavity for 5 months. The lesion had a firm texture when touched. According to the patient's parent the lesion had a relatively relapsing, remitting characteristic and its size was reduced after each course of antibiotic used for intermittent upper respiratory tract infections. On local examination, a painless lesion measuring $5 \mathrm{~cm}$ in greatest diameter with irregular, friable margin and bleeding on touch was found affecting mainly the anterior hard palate and the upper lip (Figure 1).

An initial clinical diagnosis of lymphoma and leukemia of the upper lip and hard palate in the oral cavity was made; an incisional biopsy was taken from the margin of the lesion and sent for histopathological examination. Routine hematological and biochemical investigations and the chest X-ray and CT scan did not reveal any abnormality except for reactive lymph nodes and findings suggestive of a minor beta thalassemia. Serological tests obtained for syphilis and HIV were found negative.

Despite a non-reactive PPD of $5 \mathrm{~mm}$, and a negative Ziehl-Neelsen staining for acid fast bacilli, microscopic examination revealed stratified squamous epithelium and subepithelium with presence of multiple necrotizing epithelioid cell granulomas and Langhans' type of giant cell (Figure 2). Malignancy was not seen in serial sections examined. On the basis of above findings, a primary diagnosis of tuberculosis of the oral cavity was made, which was later confirmed by PCR. Subsequent history taken after the diagnosis, revealed a history of frequent use of raw milk during infancy. In fact, the child was bottle fed with cow milk since two months of age. There was no family history of tuberculosis.

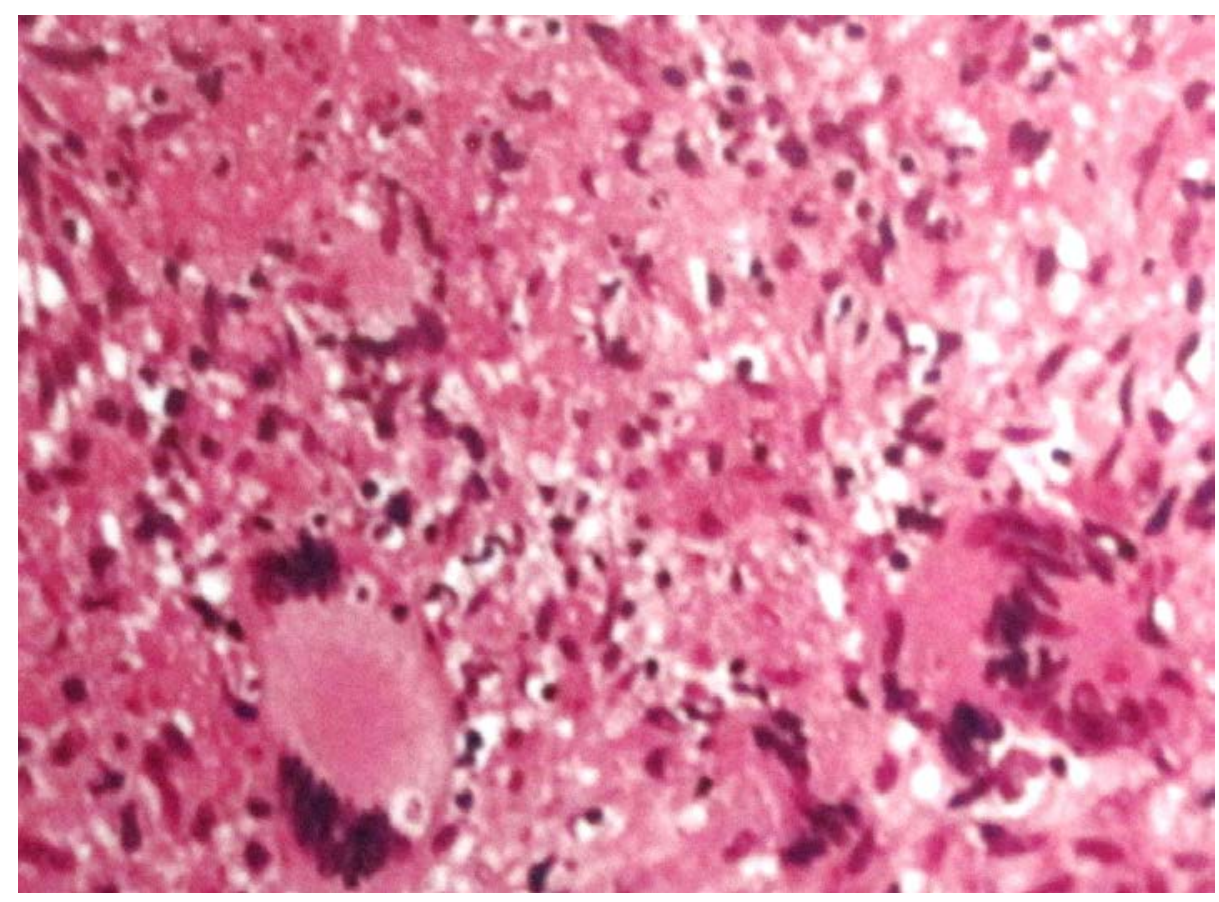

Figure 2.

The patient was treated with 2HRZE/4HR regimen of Isoniazid, Rifampicin, Ethambutol, and Pyrazinamide on alternate days thrice a week for two months, followed by Rifampicin and Isoniazid on alternate days, thrice a week for the next four months. The patient symptoms remarkably improved and he became asymptomatic within the next months.

\section{Discussion}

Because of an intact squamous epithelium of the oral mucosa which makes tuberculosis bacilli penetration difficult and provides protection against the infection, tuberculosis of the oral cavity is an uncommon occurrence. It appears that the organisms are carried most likely in the sputum and enter the mucosal tissue through a small tear in the oral mucosa. Local predisposing factors include poor hygiene, local trauma, dental extraction, leukoplakia, jaw fracture, cyst and abscess [3].

The most common sites for oral tuberculosis are tongue, gum, and palate. Other sites include the lip, cheek, uvula, and alveolar mucosa. History of consumption of unboiled raw milk and HIV infection have also been implicated. In the present case, the patient's parent gave the history of frequent use of raw milk after the histopathological diagnosis of oral tuberculosis, and chest $\mathrm{X}$-ray did not reveal any lesion. Hence, in our opinion, the lesion in present case is primary and it is caused by Mycobacterium bovis infection [4]. According to Somoskovi, et al. the negative Ziehl-Neelsen staining is a possible event in routine staining for acid fast bacilli [5].

\section{Conclusion}

Tuberculosis of the oral cavity is relatively rare and has widely become a missed diagnosis of oral lesions. Dental practitioners need to be aware that TB may occur in the oral cavity and should be considered in the differential diagnosis of any ulcerated, indurated non-healing lesion of the oral cavity especially among lower socioeconomic groups. Furthermore, efforts should be made to control oral TB by early detection and referral of the patient to a specialist for proper management. 


\section{Competing Interests}

The authors declare that they have no financial or personal relationship(s) which may have inappropriately influenced them in writing this article.

\section{References}

[1] Crompton GK, Haslett C, Chilvers ER (1999) Diseases of the respiratory system. In: Haslett Chilvers ER, Hunter JAA, Boon
NA (eds). Davidson's principles and practice of medicine (18th edn). London: Churchill Livingstone. 347-353.

[2] Pekiner FN, Erseven G, Borahan MO, Gumru B (2006) Natural barrier in primary tuberculosis inoculation: oral mucous membrane. Int J Tuberc Lung Dis 10:1418.

[3] SezerB, Zeytinoglu M, Tuncay U, Unal T (2004) Oral mucosal ulceration: a manifestation of previously undiagnosed pulmonary tuberculosis. J Am Dent Assoc135: 336-40.

[4] B.V.Karthikeyan,A.R.Pradeep,andC.G.D.Sharma (2006) Primary tuberculous gingival enlargement: a rare entityJournal of the Canadian Dental Association 72: 645-648.

[5] Somoskövi, Á., J. E. Hotaling, M. Fitzgerald, D. O’Donnell, L. M. Parsons, and M. Salfinger( 2001) Lessons from a Proficiency Testing Event for Acid-Fast Microscopy, Chest 120: 250-257. 